This document was prepared in conjunction with work accomplished under Contract No. DE-AC09-96SR18500 with the U. S. Department of Energy.

\title{
DISCLAIMER
}

This report was prepared as an account of work sponsored by an agency of the United States Government. Neither the United States Government nor any agency thereof, nor any of their employees, nor any of their contractors, subcontractors or their employees, makes any warranty, express or implied, or assumes any legal liability or responsibility for the accuracy, completeness, or any third party's use or the results of such use of any information, apparatus, product, or process disclosed, or represents that its use would not infringe privately owned rights. Reference herein to any specific commercial product, process, or service by trade name, trademark, manufacturer, or otherwise, does not necessarily constitute or imply its endorsement, recommendation, or favoring by the United States Government or any agency thereof or its contractors or subcontractors. The views and opinions of authors expressed herein do not necessarily state or reflect those of the United States Government or any agency thereof. 
REVISION: 1

KEYWORDS:

Autocatalytic Decomposition Hydrazoic Acid

AB Controls

\section{HYDRAZOIC ACID CONTROLS AND RISKS WHEN PROCESSING NEPTUNIUM SOLUTIONS IN HB-LINE PHASE II (U)}

March 11, 2004

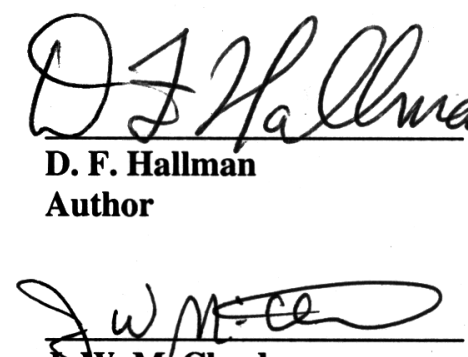

J.W. Mcclard

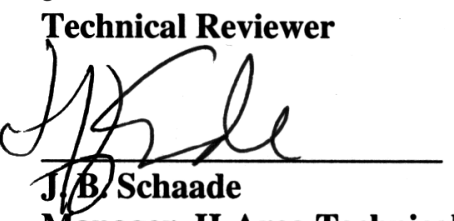

Manager, H-Area Technical Support

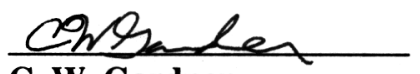

C. W. Gardner

Manager, HB-Line Engineering
Retention:

10 Years

CLASSIFICATION:

UNCLASSIFIED

Does not contain UCNI

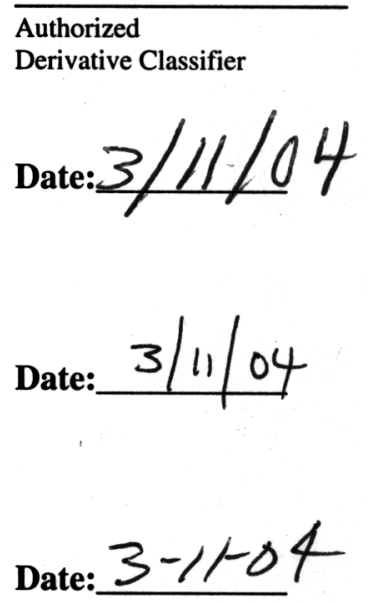

Date:3-11-04
Distribution:

C. E. Nickell, 704-2H

D. E. Alexander, 704-2H

P. R. Pritchard, 221-13H

D. W. Murdoch, 221-13H

E. R. Russell, 704-2H
R. H. Smith, 704-2H

M. N. Borders, 221-H, HBL

M. J. Lewczyk, 221-H, HBL

T. A. Bolton, 704-2H

A. J. Cappucci, 707-F
T. E. Worth, 221-H, HBL

D. M. Cato, 704-2H

J. W. Posnick, 704-2H

J. M. Low, 705-H

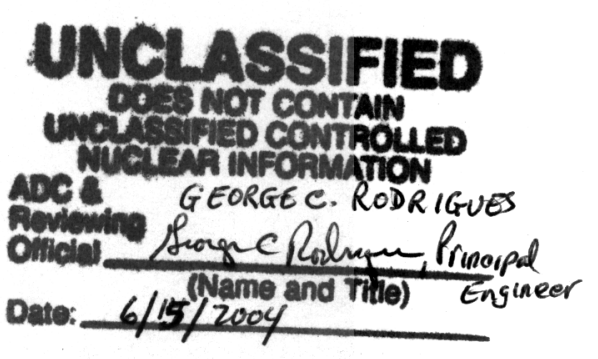




\section{Revision History}

Rev. 0, Initial Issue

Rev. 1, Issued to include partition wash as a hydrazine mononitrate-treated stream; extensive changes were made, so no revision bars are used 


\section{TABLE OF CONTENTS}

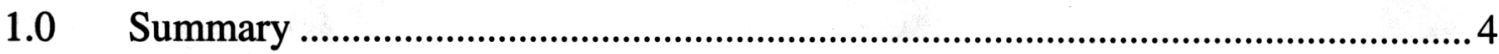

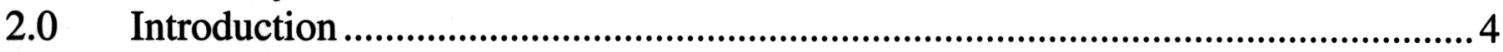

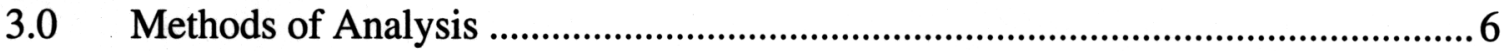

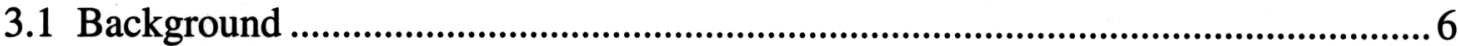

3.2 Frequency Of A Hydrazoic Acid Autocatalytic Decomposition Reaction In The Liquid Phase. .8

3.3 Frequency of a Hydrazoic Acid Autocatalytic Decomposition Reaction in the Vapor Phase...

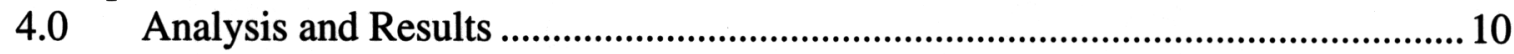

4.1 Column Feed Adjustment in JT-72 .................................................................. 10

4.2 Hydrazine Mononitrate Addition to the Partition Wash Solution........................ 12

4.3 Hydrazine Mononitrate Addition to the Resin Elutriant Solution...........................13

4.3.1 Hydrazoic Acid in the Elutriant Makeup Tank ............................................... 13

4.3.2 Hydrazoic Acid Concentration in Downstream Tanks ...................................... 15

4.4 Hydrazine Mononitrate Addition to the Precipitator Feed Adjust Tank ................. 17

5.0 Assumptions ................................................................................................ 17

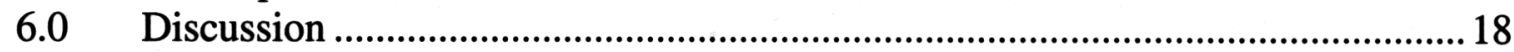

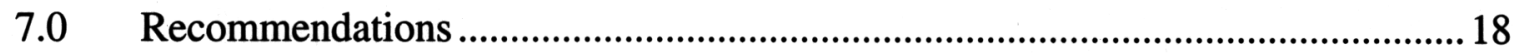

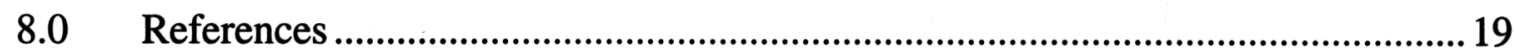




\subsection{Summary}

Hydrazine mononitrate will be used in processing neptunium in Phase II of HB-Line to scavenge nitrous acid (a byproduct of nitric acid) in the column feed adjustment tank (JT71 or JT-72), the partition wash solution, and the elutriant solution. Nitrous acid can interfere with valence adjustment chemicals (e.g., ferrous sulfamate and ascorbic acid) and thus interfere with controls that give proper neptunium valence. Hydrazine mononitrate reacts with nitrous acid to form hydrazoic acid, and the hydrazoic acid also reacts with nitrous acid. The concentration of hydrazoic acid can be controlled below the autocatalytic decomposition limit in both liquid and vapor by controlling the maximum concentration of hydrazine mononitrate in the acid solution. The concentration of nitrous acid available for reaction with hydrazine mononitrate also limits the amount of hydrazoic acid in solution. The hydrazine mononitrate concentration will be controlled at or below $0.15 \mathrm{M}$ in the neptunium solutions to maintain the hydrazoic acid concentration below autocatalytic decomposition limits. Equipment failures and/or human errors that could cause hydrazine mononitrate to be present above this limit are evaluated. Satisfying the assumptions stated in this report result in a frequency of Beyond Extremely Unlikely (BEU) for a hydrazoic acid autocatalytic decomposition reaction in either the liquid or vapor phase of the process vessels.

When Revision 0 of this document was issued, no partition wash was planned, no elutriant treatment was planned, and hydrazine mononitrate treatment in the concentrate tank was planned. The partition wash has been added to enhance separation of plutonium. Treating the elutriant with hydrazine mononitrate is now planned. Revision 1 is being issued to document that a hydrazoic acid autocatalytic reaction is still BEU under the revised flowsheet.

\subsection{Introduction}

The feed solution for neptunium processing in HB-Line Phase II is about 12 grams/liter neptunium in nitric acid solution. This solution is stored in H-Canyon Tank 8.5.

Phase II contains two parallel trains for processing neptunium solutions. Generally, the trains are operated independently. Source terms are calculated based on simultaneous operation of both trains, but this is not planned. Where two equipment designators are used below in parentheses, they refer to equipment in both trains.

For neptunium processing in HB-Line, hydrazine mononitrate use is planned in the column feed stream, the partition wash stream, and the elutriant stream. Hydrazine mononitrate is currently not planned to be used in the resin conditioning stream, the decontamination stream, or the precipitation feed adjustment tank However, an analysis of hydrazine mononitrate addition to the precipitation feed adjustment tanks (NT-41/42) is included in this report, even though hydrazine mononitrate use is not planned for these tanks. The hydrazine mononitrate residual in the elutriant is adequate to protect the 
valence state of neptunium in these tanks for normal processing. If an extended outage occurs while neptunium solution is in these tanks, and it is decided that the material in these tanks need to be recovered to support the campaign objectives, hydrazine mononitrate addition may be desirable. This report justifies the safety of hydrazine mononitrate addition to this tank at planned treatment concentrations up to $0.15 \mathrm{M}$, to maintain this option.

Neptunium solution at about 12 grams neptunium per liter will be transferred to JT-71 from Tank 8.5 in H-Canyon. From JT-71, a volume of solution containing about 8000 grams of neptunium will be transferred to JT-72. Ferrous sulfamate will be added to this solution, the mixture will be agitated, then stored for a minimum of 16 hours and a maximum of 72 hours. Nitric acid and hydrazine mononitrate will then be added to the solution. The nitrate concentration of this solution will be adjusted to $\sim 8 \mathrm{M}$ and ferrous sulfamate and hydrazine mononitrate added to adjust the neptunium valence. This solution will then be transferred to column feed tanks and to the ion exchange column. Neptunium and other actinides will be concentrated (not all actinides will be efficiently concentrated) on the resin column. Elutriant will be passed through the resin column to elute a concentrated solution of neptunium and other actinides. The elutriant will contain $0.05 \mathrm{M}$ hydrazine mononitrate for nitrous acid scavenging during eluting and also while the solution is held in the concentrate tank for further processing. This solution, containing high ( $\sim 50$ grams/liter) neptunium concentration is collected in the concentrate tanks (NT-21 and NT-22). Batches of the concentrated solution, containing about 1000 grams of neptunium, are then transferred to the feed adjust tanks (NT-41 and NT-42). This solution is heated to $50 \pm 5^{\circ} \mathrm{C}$ for in preparation for precipitation (Reference 1).

The neptunium solution will then be pumped to the precipitation tanks for precipitation with oxalic acid to form solid neptunium oxalate. A batch of the neptunium solution will first be pumped to the precipitator, then the oxalic acid will be metered into the solution. There is a small nominally four liter first stage precipitation tank (NP-1 and NP-3) that sits on top of a nominally 75 liter second stage precipitator (NP-2 and NP-4). This gives the capability to perform simultaneous feed, two stage precipitation. However, for the neptunium processing campaign, no mixing occurs in this first stage tank, and the valve on the bottom of the tank is fully opened to allow flow into the second stage tank.

The solution in the precipitator tank is stirred until precipitation is complete. This solution, now containing neptunium oxalate solids, excess oxalic acid, and any hydrazoic acid formed, is then vacuum transferred through a filter for solids separation. The liquid flows through the filter into a nominally 100-liter filtrate tank (NT-51 and NT-52). Any hydrazoic acid formed is transported with the liquid into the filtrate tank. The filtration solution will be at approximately $1.3 \mathrm{M}$ nitric with the inclusion of the "cake wash $1.4 \mathrm{M}$ nitric \& $0.1 \mathrm{M}$ oxalic" solution used during the filtration step.

Sodium permanganate is then added to the filtrate tank solution. This is added to decompose the oxalic acid excess used to ensure complete precipitation of the neptunium nitrate. It also decomposes the oxalate associated with any solid neptunium oxalate that passes through the filter. The sodium permanganate also oxidizes any hydrazoic acid that 
is present, and any hydrazine mononitrate residual that is present. Sodium nitrite is then added, if necessary, to dissolve any manganese dioxide formed from the sodium permanganate addition. Since any hydrazine mononitrate residual had been destroyed by the permanganate, no hydrazoic acid is formed from the nitrite addition.

\subsection{Methods of Analysis}

The method of formation of hydrazoic acid while processing neptunium solutions in Phase II of HB-Line is considered in deriving controls to prevent (decrease the frequency of) a hydrazoic acid autocatalytic decomposition. The frequency of a hydrazoic acid autocatalytic decomposition reaction is evaluated considering the failures required to reach conditions where an explosion can potentially occur and the credible locations of a hydrazoic acid autocatalytic decomposition. The controls that reduce the consequences and frequency of a hydrazoic acid autocatalytic decomposition reaction in HB-Line for processing neptunium solutions are identified in the individual evaluations, then summarized in the Assumptions section to enhance ease of identification of the controls.

Background information on the formation of hydrazoic acid, and the mechanism for formation of an autocatalytic decomposition mixture, is discussed further below, to establish the basis for the controls selected to prevent a hydrazoic acid explosion. The methods used to evaluate the frequency of a hydrazoic acid autocatalytic decomposition reaction in Phase II of HB-Line, and the controls to prevent this, are also discussed below.

\subsection{Background}

The reasons for hydrazine mononitrate usage as a chemical additive are discussed above. Hydrazine mononitrate requires special handling during addition to minimize personnel exposure, but this is a common chemical hazard and is controlled through the WSRC Chemical Control Program (Reference 2) and will not be discussed further in this Technical Report. Hydrazine mononitrate is not a processing hazard except for its contribution to hydrazoic acid formation.

Hydrazine mononitrate reacts with nitrous acid $\left(\mathrm{HNO}_{2}\right)$ to produce two molecules of water and a molecule of hydrazoic acid $\left(\mathrm{HN}_{3}\right)$, as shown in Equation 1 (Reference 3).

$$
\mathrm{HNO}_{2}+\mathrm{N}_{2} \mathrm{H}_{4} \longrightarrow \mathrm{HN}_{3}+2 \mathrm{H}_{2} \mathrm{O} \quad \text { Equation } 1
$$

Hydrazoic acid also reacts with nitrous acid to produce water and gaseous products, as shown in Equation 2.

$$
\mathrm{HNO}_{2}+\mathrm{HN}_{3} \longrightarrow \mathrm{N}_{2} \mathrm{O}+\mathrm{N}_{2}+\mathrm{H}_{2} \mathrm{O} \quad \text { Equation } 2
$$

The equilibrium amount of hydrazoic acid in solution depends on the relative rates of Equations 1 and 2. Laboratory experiments show that, because of the reaction of 
hydrazoic acid with nitrous acid (Equation 2 above) the maximum equilibrium

concentration of hydrazoic acid in liquid is about $25 \%$ of the hydrazine mononitrate concentration. At higher hydrazine mononitrate concentrations $(0.19 \mathrm{M}$ and $0.44 \mathrm{M})$ the maximum hydrazoic acid concentration in the liquid was $12 \%$ of the hydrazine mononitrate concentration (Reference 3 ). In this evaluation, it is conservatively assumed the hydrazoic acid concentration can temporarily be as large as $1 / 3$ the hydrazine mononitrate value. Therefore, if the hydrazine mononitrate concentration in solution is controlled, the hydrazoic acid concentration is controlled.

It is reported (Reference 5) that solutions must contain at least 30 weight $\%(\sim 7.3 \mathrm{M})$ hydrazoic acid before an explosion can occur in liquid. Therefore, if the concentration of hydrazine mononitrate used for nitrous acid scavenging is limited below $(7.3 * 3 \sim 21.9 \mathrm{M})$ an explosive mixture in liquid cannot form.

Equation 1 shows that one mole of hydrazine mononitrate produces one mole of hydrazoic acid and consumes one mole of nitrous acid. The hydrazoic acid then consumes one mole of nitrous acid in Equation 2. From experimental evidence, the ratio of the concentration of hydrazoic acid to hydrazine mononitrate varies significantly, peaking at 0.25 , or $1 / 4$. For this evaluation, a ratio of $1 / 3$ is chosen; this is a conservative ratio for this evaluation. Equation 1 shows that one mole of nitrous acid is consumed to produce one mole of hydrazoic acid. Equation 2 then consumes $2 / 3$ of a mole of nitrous acid is consumed to produce $1 / 3$ mole of hydrazoic acid. To achieve $1 / 3$ mole of hydrazoic acid at equilibrium, 5/3 mole of nitrous acid must be consumed. Therefore, five moles of nitrous acid and three moles of hydrazine mononitrate are consumed per mole of hydrazioc acid that remains in solution.

Hydrazoic acid is relatively volatile (boiling point $\sim 37^{\circ} \mathrm{C}$ ) and is present in the vapor above a liquid solution of hydrazoic acid. Hydrazoic acid may undergo a rapid autocatalytic decomposition in the vapor phase. The partial pressure of hydrazoic acid in the vapor phase where decomposition may occur is 65 torr (Reference 5). There are 760 torr in an atmosphere; therefore, the volume percent at which hydrazoic acid can decompose is $(65 / 760) \sim 8.5 \%$, or 0.085 atmospheres. This coefficient decreases as nitric acid concentration increases and as temperature decreases, so the use of the above bounds the operating conditions. Using a bounding Henry's Law coefficient bounds the vapor phase hydrazoic acid concentration. The amount of vapor phase hydrazoic acid that exists in equilibrium above a hydrazoic acid solution is given by the following equation:

$$
\mathrm{y}_{\mathrm{HN} 3}=\mathrm{H}_{\mathrm{HN} 3}{ } \mathrm{x}_{\mathrm{HN} 3}
$$

Equation 3

Where:

$\mathrm{y}_{\mathrm{HN} 3}=$ partial pressure of hydrazoic acid in vapor phase, atmospheres $\mathrm{H}_{\mathrm{HN} 3}=$ Henry's Law coefficient for hydrazoic acid, atmospheres/(moles/liter) $\mathrm{x}_{\mathrm{HN} 3}=$ concentration of hydrazoic acid in liquid phase, moles/liter

All tanks are at ambient temperature, except for the feed additive tanks (NT-41/42) and the precipitate tanks (NP-2/4). These tanks are heated to $50 \pm 5^{\circ} \mathrm{C}$ (Reference 1). 
However, to provide additional operating flexibility and conservatism, the temperature of these tanks will be assumed to be $65^{\circ} \mathrm{C}$.

Reference 6 provides an equation to calculate the Henry's Law coefficient for hydrazoic acid. The equation assumes that no nitric acid is present; this provides the maximum values for the Henry's Law coefficients. The hydrazoic acid Henry's Law coefficient is 0.1 atmosphere-liters/mole at $30^{\circ} \mathrm{C}$, and is 0.37 atmosphere-liter/mole at $65^{\circ} \mathrm{C}$.

The liquid concentration of hydrazoic acid that gives a decomposition partial pressure of hydrazoic acid in the vapor phase is thus $(0.085 / 0.1) \sim 0.85 \mathrm{M}$ for tanks at $30^{\circ} \mathrm{C}$ and $(0.085 / 0.37) \sim 0.23 \mathrm{M}$ in NT-41/42 and NP-2/4.

\subsection{Frequency Of a Hydrazoic Acid Autocatalytic Decomposition Reaction In The Liquid Phase}

HB-Line uses a nominally 30 weight $\%$ hydrazine mononitrate $\left[\mathrm{N}_{2} \mathrm{H}_{4} \cdot \mathrm{HNO}_{3}\right]$ solution in water for valence adjustment. Per the flowsheet, this hydrazine mononitrate concentration is $3.63 \mathrm{M}$. Decomposition reactions in liquid do not occur below $7.3 \mathrm{M}$ hydrazoic acid concentration in the liquid. Since the maximum hydrazoic acid concentration in liquid is $1 / 3$ of the hydrazine mononitrate concentration, the hydrazine mononitrate concentration that could lead to a hydrazoic acid autocatalytic reaction is $\left(7.3^{*} 3\right) \sim 21.9 \mathrm{M}$. This molarity cannot be practically attained in solutions treated with hydrazine mononitrate, since the hydrazine mononitrate is diluted by the solution it is added to. Thus the maximum achievable hydrazine mononitrate concentration is $3.63 \mathrm{M}$. This is far below the limit of $21.9 \mathrm{M}$ hydrazine mononitrate at which an autocatalytic decomposition concentration of hydrazoic acid in liquid might occur. Therefore, by limiting the hydrazine mononitrate supply to be added for nitrous acid scavenging to 30 weight $\%$ hydrazine mononitrate, HB-Line stays about a factor of six below the hydrazine mononitrate molarity that could cause explosive concentrations of hydrazoic acid in the liquid, even with uncontrolled addition.

The hydrazine mononitrate drums are delivered sealed, and remain sealed until needed. When needed, a drum pump is inserted in the drum for transferring chemicals. Hydrazine mononitrate is a salt, and the solution could increase in concentration by evaporation of water from the hydrazine mononitrate solution. This is not seen as a significant effect, since there will be no openings to the atmosphere during storage or use. The drum pump effectively inhibits evaporation of the water.

The hydrazine mononitrate is contained in drums, and is not hard-piped into the system. There is no mechanism where mechanical failure can cause inadvertent transfer of hydrazine mononitrate from the drums into the process tanks. The only mechanism for adding more hydrazine mononitrate than planned to the process tanks is human error.

The possibility of hydrazoic acid being retained on the resin column, and eluting as a more concentrated solution when the neptunium is eluted was considered. Hydrazoic 
acid is a weakly ionized acid; nitric acid is a strongly ionized acid. In strong acid solutions such as the column feed solution (8M) and the partition wash $(6.4 \mathrm{M})$, very little ionization of hydrazoic acid is expected. Further, the azide ions would have to compete with the nitrate ions for resin sites. Since nitric acid is strongly ionized, the concentration of nitrate ions, at this nitric acid concentration, will be high relative to the azide ions. It is unlikely that a significant amount of azide ion would be retained on the resin. It is also unlikely that any azide ion deposited on the resin would be eluted with the weaker nitric acid solution $(0.17 \mathrm{M})$ used to elute the neptunium from the resin. However, tests were run to determine if hydrazoic acid concentrates on the resin (Reference 7). These tests were run at $8 \mathrm{M}$ nitric acid concentration. The tests showed no concentration of hydrazoic acid on the resin. Tests were not run for the $6.4 \mathrm{M}$ nitric acid solution used for the partition wash. No concentration of hydrazoic acid on the resin would be expected from the 6.4M solution, since both nitrate and hydrogen ion concentration in this solution are still very high..

Hydrazoic acid can also concentrate in condensate if the condensing temperature is lower than the evaporation temperature. In HB-Line Phase II, the pertinent condensation point would be the vessel vent system. The vessel vent system is connected to all the Phase II vessels that contain process solutions, and a vacuum is maintained on this system to draw vapors from the vessels for cleanup before discharging the vessel vent system. The concentration of hydrazoic acid in the vapor phase increases as the solution temperature increases, and the concentration in the condensed liquid increases as the condensation temperature is lowered. The hydrazoic acid concentration in condensate in Phase II of HB-Line was evaluated using extreme conditions of evaporation at $50^{\circ} \mathrm{C}$, condensing at $0{ }^{\circ} \mathrm{C}$, and $0.15 \mathrm{M}$ hydrazoic acid in the liquid phase (Reference 6). The resulting concentration in the condensing vapor is $1.27 \mathrm{M}$ hydrazoic acid. This is also well below the explosive concentration of $7.3 \mathrm{M}$ in liquid. This concentration could only practically exist on the walls of vessel vent system piping prior to entry to the vent catch tank. This tank contains about 100 liters of water and would dilute the small amounts of hydrazoic acid transported through the vessel vent system. Further, there is no source term, other than trace levels of radioactivity, in the vessel vent system tubing and tanks, so the consequences of a liquid autocatalytic decomposition reaction would be very low. No additional controls will be implemented to control this small risk; controls that make the autocatalytic decomposition reaction in process vessel liquid and vapor BEU are adequate to control this small risk.

\subsection{Frequency of a Hydrazoic Acid Autocatalytic Decomposition Reaction in the Vapor Phase}

Hydrazoic acid can achieve the concentration to undergo a decomposition reaction in the vapor phase if the hydrazoic acid concentration in the liquid phase exceeds $0.85 \mathrm{M}$ for tanks at $30^{\circ} \mathrm{C}$ and $0.23 \mathrm{M}$ in the tanks at $65^{\circ} \mathrm{C}$. It is conservatively assumed that the concentration of hydrazoic acid in the liquid phase can be as high as $1 / 3$ of the hydrazine mononitrate concentration, if the nitrous acid supply is sufficient to support this amount of hydrazoic acid being created. Therefore, if the hydrazine mononitrate concentration is 
kept less than $\left(0.85^{*} 3\right) \sim 2.55 \mathrm{M}$ in tanks with temperatures $30^{\circ} \mathrm{C}$ or less, or $\left(0.23^{*} 3\right) \sim$ $0.69 \mathrm{M}$ in tanks with $60^{\circ} \mathrm{C}$ or less, the concentration in the vapor phase of these tanks will be less than the decomposition concentration.

\subsection{Analysis and Results}

The volumes of solutions for concentrating neptunium on the columns and partitioning the plutonium are from the flowsheet (Reference 1). They are summarized below. Inadvertent additions of hydrazine mononitrate, above that specified by procedure, will be considered to determine if hydrazoic acid concentrations that could result in autocatalytic decomposition reactions are credible.

Table 1: Volumes of Solution Added to Resin for a 2000 Gram Loading

\begin{tabular}{|l|l|l|}
\hline Stream & Column Feed & Partition Wash \\
\hline Volume, Liters & 256 & 228 \\
\hline
\end{tabular}

The volumes of the elutriant streams used are summarized below. These volumes are from Reference 1.

Table 2: Volumes of Solution in Elution Streams for a 2000 Gram Elution

\begin{tabular}{|l|l|l|l|}
\hline Stream & Heads Cut & Hearts Cut & Tails Cut \\
\hline Volume, Liters & 45 & 33.1 & 40(once per 4 column runs) \\
\hline
\end{tabular}

As stated above, the frequency of a hydrazoic acid decomposition event in liquid is BEU if the hydrazine mononitrate feed solution is $30 \%$ or less (by weight) hydrazine mononitrate. The frequency of hydrazoic acid decomposition reactions in the vapor phase from treating neptunium streams with hydrazine mononitrate is evaluated below.

\subsection{Column Feed Adjustment in JT-72}

Hydrazine mononitrate is added to the solution in JT-72 to assist in valence adjustment in the column feed solution. The flowsheet (Reference 1) specifies treating the column feed solution to achieve $0.02 \mathrm{M}$ hydrazine mononitrate. This report will evaluate a treatment concentration of $0.15 \mathrm{M}$ hydrazine mononitrate for additions to JT-72. This will provide operational margin in case it is found desirable to increase the hydrazine mononitrate concentration in this stream in the future, and to provide a bounding limit for the analysis.

As previously discussed, a series of tests were performed to determine if hydrazoic acid could be concentrated by the ion exchange resin during processing. The report (Reference 7) concluded that there will be no concentration of hydrazoic acid on the resin. This is as expected. Nitric acid is a strong acid and will ionize readily. Hydrazoic acid is a weak acid and does not readily ionize. There will be a large number of nitrate ions competing for resin exchange sites with a small number of azide ions. Also, if any 
azide ions adhere to the resin in a strong nitric acid solution, they are unlikely to be displaced from the resin with a weak elutriant (about $0.17 \mathrm{M}$ nitrate).

These tests were run at $8 \mathrm{M}$ nitric acid. The partition wash stream is at $6.4 \mathrm{M}$ nitric acid. This relatively small difference in nitric acid strength will have little effect on the behavior of hydrazioc acid relative to ionization and retention on the resin bed.

The temperature in JT-72 could be slightly above ambient due to the addition of $14 \mathrm{M}$ acid to the process solution to raise the nitrate concentration of the solution. The Henry's Law coefficient of 0.1 atmospheres/(moles/liter) is for $30^{\circ} \mathrm{C}$ in water, per Table 1 of Reference 6, and this value was used in the JT-72 analysis. This table also shows that the Henry's Law coefficient decreases as the nitric acid concentration in the solution increases. The JT-72 solution is adjusted to $\sim 8 \mathrm{M}$ nitric acid. The effect of the higher nitric acid concentration in JT-72 will compensate for the effect of any small increase of temperature above $30^{\circ} \mathrm{C}$.

Although the analysis was performed assuming JT-72 is the tank where the column feed solution is adjusted, the results would be identical had JT-71 been used as the column feed adjustment tank.

Hydrazine mononitrate treatment of JT-72 is performed by adding it to the Cold Feed Prep tank used to add the high-molarity acid needed to adjust the JT-72 solution to $8 \mathrm{M}$ nitrate. These are tanks $\mathrm{CN}-24$ or $\mathrm{CN}-25$. The flowsheet (Reference 1) shows 307 liters of nitric acid added to JT-72. Solution is pumped from these tanks through an eductor with the eductor suction valve closed for transfer to JT-72. The pump is automatically cut off when the level reaches an indicated level of 3\%. This is about 74 liters for CP-25 (higher for CP-24). The 307 liters of nitric acid is added to this existing level, to give a level of 381 liters.

The tanks contain a 2-inch overflow port (Reference 9). The centerline of this overflow port is about four inches from the top of the tank (Reference 10). The overflow port is nominally at $100 \%$ of tank span and is 553.93 liters for $\mathrm{CN}-24$ and 562.16 liters for $\mathrm{CN}$ 25 (Reference 14). The volume that can be occupied by liquid is thus $(562-381) \sim 181$ liters. This is the maximum volume that the tank holds between the pump cutoff and the overflow point

These tanks have a high-level alarm below the overflow point (normally set at $90 \%$ of span). This alarm annunciates in the continuously-occupied control room. To reach the overflow point, the alarm would have to be ignored by the personnel in the control room. Continued additions after the alarm setpoint is reached are unlikely, even though assumed in this analysis.

If, through human errors, hydrazine mononitrate was added to the tanks until the overflow port was reached, this would be $(181 * 3.63) \sim 657$ moles of hydrazine mononitrate. The concentration of hydrazine mononitrate would be $(657 / 562) \sim 1.17 \mathrm{M}$ hydrazine mononitrate. The maximum hydrazoic acid that could form is $(1.17 / 3) \sim$ 
0.39M. Applying Equation 3 with a Henry's Law coefficient of 0.1, the partial pressure in air would be $\sim 30$ torr. This is well below the 65 torr where there may be an autocatalytic reaction in vapor.

The hydrazine mononitrate solution is miscible with nitric acid, so additions after the overflow point are reached would mix somewhat with the solution in the tank before overflowing. This could cause a slow concentration increase in the hydrazine mononitrate concentration in the 600-liter tanks. This is not a significant factor for this analysis. The conservative assumption that the concentration of hydrazoic acid can be $1 / 3$ of the hydrazine mononitrate concentration, rather than the maximum concentration of $1 / 4$ shown by the data, more than compensates for the slow increase of hydrazine mononitrate concentration that would occur during tank overflow.

\subsection{Hydrazine Mononitrate Addition to the Partition Wash Solution}

After loading the column, the column will be washed with a solution to partially remove the plutonium from the column. This solution will be $6.4 \mathrm{M}$ nitric acid, $0.05 \mathrm{M}$ ferrous sulfamate, and $0.05 \mathrm{M}$ hydrazine mononitrate (Reference 1 ). This analysis will be based on a specification of $0.15 \mathrm{M}$ hydrazine mononitrate in this solution, to allow future operational flexibility if needed.

The flowsheet specifies a partition wash volume of 228 liters. Tank CN-22 in Cold Feed Prep is the tank planned to be used for making up the partition wash solution. It is conservative to use the parameters for the 600-liter tank with the largest volume between $3 \%$ (pump cutoff) and $100 \%$ (overflow). This is $\mathrm{CN}-25$, with a volume of 487 liters (Reference 14).

Two charges will be made up per tank. The volume of the final treated solution, with $0.05 \mathrm{M}$ hydrazine mononitrate in it, is 228 liters. The volume of hydrazine mononitrate added was $(228 * 0.05 / 3.63) \sim 3$ liters, so the volume with no hydrazine mononitrate is 225 liters. For two charges, the volume before hydrazine mononitrate addition is 450 liters. Applying equation 4 with a final hydrazine mononitrate concentration of $0.15 \mathrm{M}$, the volume of hydrazine mononitrate to be added is 20 liters, for a total wash solution volume of 470 liters. When the pump cutoff volume (75 liters) is added to this, the total volume in the tank is 545 liters, or above the $96 \%$ level of the tank.

If it is necessary to increase the hydrazine mononitrate concentration to $0.15 \mathrm{M}$ in the partition wash, the high alarm point would have to be set at about $97 \%$ to avoid actuating the alarm during normal operations. Assuming one charge is made up at a time is a more conservative case for bounding the potential hydrazoic acid concentration as a result of human errors.

For a single partition wash batch, the volume of nitric acid and ferrous sulfamate is 225 liters. Applying equation 4, 10 liters of hydrazine mononitrate is needed to achieve a $0.15 \mathrm{M}$ solution, bringing the solution volume to 235 liters. Adding the pump heel for 
$\mathrm{CN}-25$ (75 liters), the total volume in $\mathrm{CN}-25$ would be 310 liters. The overflow is at 562 liters, so $(562-310) \sim 252$ liters of hydrazine mononitrate solution could be added before overflow. The hydrazine mononitrate concentration in this solution would be $(3.63 * 252 / 562) \sim 1.63 \mathrm{M}$. The potential hydrazoic acid concentration would be $(1.63 / 3) \sim$ $0.54 \mathrm{M}$. Applying equation 3 with a Henry's Law coefficient of 0.1 , the hydrazoic acid concentration in vapor would be $\sim 41$ torr, well below 65 torr. Therefore, preparation of the partition wash solution can not result in hydrazoic acid concentrations that could cause an autocatalytic decomposition event.

\subsection{Hydrazine Mononitrate Addition to the Resin Elutriant Solution}

Hydrazine mononitrate is added to the elutriant (the stream that elutes the neptunium from the resin bed). The flowsheet (Reference 1) specifies a concentration of $0.17 \mathrm{M}$ nitric acid for elutriant. The maximum molarity that can be expected to elute neptunium from the columns is $4 \mathrm{M}$, and the nitrate molarity in the concentrate tank is $2.5 \mathrm{M}$ (Reference 1). The nitric acid strength in the elutriant tank is $0.17 \mathrm{M}$.

Two cases are considered below. Case 1 considers the hydrazoic acid concentration in the tank used to make the solution up. Case 2 considers the hydrazoic acid concentrations in the Phase II tanks downstream of the resin columns.

\subsubsection{Hydrazoic Acid in the Elutriant Makeup Tank}

The low concentration of nitric acid in the elutriant tank limits the amount of nitrous acid that could be present. The maximum amount of nitrous acid that could be present is $0.17 \mathrm{M}$; this could occur if all the nitric acid were reduced to nitrous acid. Nitrous acid can be formed from nitric acid by radiation or light. Fresh nitric acid is diluted with nonradioactive process water in the Cold Feed Preparation tanks. The acid was stored in metal tanks prior to transfer to Cold Feed Prep. It was not exposed to light during storage. Recycled nitric acid is not used in HB-Line. The expected level of nitrous acid in fresh nitric acid is in the ppm range; a nitrous acid concentration of $1 \%$ is considered high. However, for purposes of a conservative evaluation of conditions in the Cold Feed Prep tank containing the elultriant solution, it will be assumed that, by error, the tank contains $14 \mathrm{M}$ nitric acid and further errors occur during hydrazine mononitrate addition. Note that this effect would be limited to the elutriant feed tank; the refractometers would prevent this solution being fed to the column.

It is planned to use Tank $\mathrm{CN}-23$ to contain the elutriant solution, but this analysis applies to tanks CN-22, CN-24, and CN-25. All these tanks are as described in Reference 10. This analysis uses the level in the tank at the indicated level of $3 \%$ as an input. Of the four tanks, CN-25 has the largest volume between the pump heel and the overflow (Reference 14). This volume is 487 liters. Choosing the tank with the lowest volume is conservative for this evaluation. 
Further, the current flowsheet (Reference 1) specifies a heads cut of 45 liters and a hearts cut of 33.1 liters per column run, with a single tails cut of 40 liters after four column runs. This is with current goals of efficiency for the column loading and eluting. This analysis will consider multiple erroneous additions of hydrazine mononitrate to the elutriant tank in addition to the error in making up the correct acid strength. It will show that the tank cannot hold sufficient hydrazine mononitrate to generate hydrazoic acid at autodecomposition concentrations. Therefore, lower volumes of elutriant bound the values currently in Reference 1 . If a 1500 gram batch were to be processed through the resin column, the volumes for the heads cut, and hearts cut, would probably decrease to the values shown in Table 4 below. The values chosen for a bounding analysis are shown in Table 4 below.

Table 3: Elutriant Solution Volumes

\begin{tabular}{|l|l|l|}
\hline Elution Step & Volume per cycle, liters & $\begin{array}{l}\text { Volume for four column } \\
\text { runs, liters }\end{array}$ \\
\hline & & \\
\hline Head Cut & 35.3 & 130 \\
\hline Hearts Cut & 30 & 120 \\
\hline Tails Cut & 40 & 40 \\
\hline TOTAL & & 290 \\
\hline
\end{tabular}

Again using $\mathrm{CN}-25$ as the bounding tank, the total volume of hydrazine mononitrate that could be added to this tank is (487- 290) 197 liters. The hydrazine mononitrate concentration would be $(3.63 * 197 / 562) \sim 1.18 \mathrm{M}$, and the potential hydrazoic acid concentration would be $(1.18 / 3) \sim 0.39 \mathrm{M}$. Applying equation 3 with a Henry's Law coefficient of 0.1 , the resulting partial pressure of hydrazoic acid in the vapor space is 30 torr, which is well below the autocatalytic decomposition pressure of 65 torr.

These tanks have a high-level alarm that actuates in the continuously-occupied control room below the overflow point. To reach the overflow point, this alarm would have to be ignored by the personnel in the control room. Continued additions after the alarm setpoint is reached are unlikely, even though assumed in this analysis.

After the overflow point is reached, there would be some mixing of the added hydrazine mononitrate solutions with the solution already in the tank, so the hydrazine mononitrate concentration in the tank would slowly increase. This is not a significant factor for this analysis. The conservative assumption that the concentration of hydrazoic acid can be $1 / 3$ of the hydrazine mononitrate concentration, rather than the maximum concentration of $1 / 4$ shown by the data, more than compensates for the slow increase of hydrazine mononitrate concentration that would occur during tank overflow.

Since the passive overflow prevents addition of enough hydrazine mononitrate to reach autocatalytic decomposition concentrations in either liquid or vapor phase, the frequency of a hydrazoic acid explosion as a result of human errors when adding hydrazine mononitrate to the elutriant solution is BEU. 
Since hydrazoic acid does not concentrate on the resin, and the concentrate tanks (JT21/22) are not heated, the concentration of hydrazoic acid in the concentrate tanks will not be higher than the concentration in the elution feed tanks. Hydrazoic acid concentrations in these tanks will be below the concentration where autocatalytic decomposition events can occur.

\subsubsection{Hydrazoic Acid Concentration in Downstream Tanks}

The solution is then fed forward from the concentrate tanks into the precipitator feed adjust tanks. Reference 1 does not specify any chemical additions in this tank; past operating experience shows that, if the eluent stream is treated to $0.05 \mathrm{M}$ hydrazine mononitrate, adequate hydrazine mononitrate is present in the chemical addition tank.

The solution then is heated to $50 \pm 5^{\circ} \mathrm{C}$ in preparation for precipitation. For this analysis, a bounding temperature of $65^{\circ} \mathrm{C}$ is assumed.

The refractometers will not allow feed of nitric acid higher than $9 \mathrm{M}$ to the columns. Further, feed will not be diverted from the raffinate stream (to H-Canyon) until a colorimeter detects the presence of $\mathrm{Np}$ in the solution. The elutriant solution is $0.17 \mathrm{M}$ nitric acid, but neptunium will start eluting from the column at about $4 \mathrm{M}$ nitric acid. The concentration in the hearts cut will be a mixture of the $8 \mathrm{M}$ nitric acid solution used for the column decontamination wash and the $0.17 \mathrm{M}$ nitric acid solution used for elution. If the molarity of the elutriant solution is too high, neptunium will not elute and the highmolarity solution will not be diverted to the concentrate tanks.

Per reactions 1 and 2, hydrazoic acid is not formed once nitrous acid is exhausted. Nitrous acid is a byproduct in nitric acid; it can be formed by radiation or light. The nitric acid used in HB-Line is "fresh" acid; recycled acid is not used. The nitric acid is stored and blended in metal tanks, so it is only incidentally exposed to light. It would be exposed to radioactivity from the neptunium solution after elution. The neptunium solutions contain fewer curies than do plutonium solutions, due to the long half-life of neptunium -237 (2,144,000 years). The energy emission rate is much less than for plutonium solutions. The energy emission rate is about three orders of magnitude lower for the neptunium solutions in H-Canyon Tank 8.5 than for heat source solutions encountered in the past. The nitrous acid formation rate from radioactive materials emissions will be low for the neptunium solutions.

A very conservative estimate is that the nitrous acid concentration may be as high as $1 \%$ of the nitric acid concentration. As previously discussed, the nitric acid used in HB-Line is "new" (rather than recycled) acid, has been protected from light, and has low levels of energy emission from radioactive materials. Further, operating experience with the eluent treated to $0.05 \mathrm{M}$ hydrazine mononitrate shows that there is still adequate hydrazine mononitrate in the precipitator adjust tanks (NT-41/42) to protect the Np against valence changes reinforces that the nitrous acid concentration of this stream is 
low. If significant nitrous acid had been present, the hydrazine mononitrate would have been depleted.

From the discussion in Section 3.1 of this document, five moles of nitrous acid (and three moles of hydrazine mononitrate) are consumed to achieve one mole of hydrazoic acid. As a bounding assumption, it will be assumed that the nitrous acid concentration is $10 \%$ of the nitric acid concentration in the concentrate tanks. From Reference 1, this concentration is $2.5 \mathrm{M}$ nitric acid. Again for a bounding case, it will be assumed the nitric acid concentration is $4.0 \mathrm{M}$, the concentration at which neptunium may begin eluting from the column. The nitrous acid concentration would be $(0.10 * 4.0) \sim 0.4 \mathrm{M}$ nitrous acid. The hydrazoic acid concentration in the liquid would be $(0.4 / 5) \sim 0.08 \mathrm{M}$, assuming hydrazine mononitrate had been added to a concentration of at least $0.24 \mathrm{M}$. The vapor phase concentration in the concentrate tanks would then be $((0.08 * 0 * 1 * 760) \sim 6$ torr, well below the 65 torr where an autocatalytic decomposition reaction may occur.

An aliquot of this solution containing about 1000 grams of neptunium will then be moved to the Feed Adjust Tanks (JT-41/42). The solution will then be heated so it can be precipitated while hot. It is assumed that the solution will be heated to $65^{\circ} \mathrm{C}$, to bound planned operations. The Henry's Law coefficient at this temperature is 0.37 liters/atmosphere-mole. Since this is the same solution that was evaluated in the concentrate tanks, the hydrazoic acid concentration in the liquid is the same as discussed above for the concentrate tanks. The hydrazoic acid partial pressure in the vapor, if the liquid is at $65^{\circ} \mathrm{C}$, is $\left(0.08^{*} 0.37^{*} 760\right) \sim 23$ torr. This is far below the 65 torr partial pressure needed for an autocatalytic decomposition reaction.

The hot solution is next sent to the precipitators (NP-2 and NP-4). Oxalic acid is then metered into the solution with stirring, to form neptunium oxalate. Heat is added to maintain the temperature, but the temperature does not go above $65^{\circ} \mathrm{C}$. The hydrazoic acid concentration in the liquid is being continuously diluted with the oxalic acid during precipitation, so the concentrations in both liquid and vapor are bounded by the analysis for the Feed Adjustment Tanks.

The solution containing neptunium oxalate is then filtered. Any hydrazoic acid in the solution is sent to the filtrate tanks (NT-51/52). These are unheated tanks, so the concentration in the liquid and vapor are bounded by the conditions assumed above for the Feed Additive Tanks. Permanganate is then added to oxidize the excess oxalate in solution. The permanganate also oxidizes any hydrazoic acid and hydrazine mononitrate present.

In summary, an autocatalytic decomposition reaction in tanks NT/21/22, NT-41/42, NP$2 / 4$, and NT-51/52 resulting from erroneous hydrazine mononitrate additions to the elutriant solution is BEU. 


\subsection{Hydrazine Mononitrate Addition to the Precipitator Feed Adjust Tank}

Adjustment chemicals are added, as necessary, to the feed adjust tank (NT-41/42) to ensure proper precipitation. The flowsheet (Reference 1) assumes no chemical addition will be needed, unless solutions are left in the feed adjust tank for greater than two weeks before precipitating. It assumes there will be sufficient residual hydrazine mononitrate in the eluted solution to preclude the need for more additions. Because of the potential problems with ascorbic acid use, it is more likely that aged solutions awaiting precipitation would be discarded rather than precipitated. However, as stated earlier in this report, the effects of adding hydrazine mononitrate to NT-41/42 will be analyzed here to preserve the option of future addition.. For this analysis, it will be assumed that hydrazine mononitrate is added to NT-41/42 to achieve a concentration of $0.15 \mathrm{M}$.

NT-41/42 can hold about 77 liters of solution (Reference 11). It will receive about 26 liters of solution, at a nitric acid concentration of about 2.3M (Reference flowsheet), from the concentrate tank.

Previously, an autocatalytic decomposition reaction in NT-41/42 due to errors in adding excessive hydrazine mononitrate to the elutriant solution was evaluated. The evaluation discussed here is for excessive hydrazine mononitrate added directly to solutions in NT41/42. The same principles are applicable to excessive hydrazine mononitrate added directly to this tank. The bounding nitric acid concentration in this tank is $(0.01 * 4) \sim$ $0.4 \mathrm{M}$. Since five moles of nitrous acid are consumed to produce one mole of hydrazioc acid (see preceding discussion) the bounding hydrazoic acid concentration is $(0.4 / 5) \sim$ $0.08 \mathrm{M}$ in the liquid. This is fare below the $7.3 \mathrm{M}$ limit for an autocatalytic decomposition reaction in liquid. The contents of this tank are heated so the solution will be hot during precipitation; a bounding temperature of $65^{\circ} \mathrm{C}$ is assumed. The maximum partial pressure of hydrazoic acid in vapor, using a Henry's Law coefficient of 0.37 atmospheres-liter/mole, is $(0.08 * 0.37 * 760) \sim 23$ torr. This is far below the partial pressure of 65 torr where an autocatalytic decomposition reaction in vapor is possible. Therefore, an autocatalytic decomposition reaction from adding excessive hydrazine mononitrate to NT-41/42 is BEU.

\subsection{Assumptions}

1 Liquid volumes for streams treated with hydrazine mononitrate are as specified in Reference 1.

2 The volumes of solutions used for this evaluation assume that four consecutive column runs of about 2000 grams each are made, and a tails cut is used only at the end of the fourth run.

3 The maximum strength of hydrazine mononitrate used for feed adjustment is 3.63M. The site plans to purchase $3.63 \mathrm{M}$ hydrazine mononitrate. 
4 The amount of hydrazine mononitrate to be added to a tank will be specified by procedure.

$5 \quad$ Hydrazine mononitrate must be manually transported to the addition stations. HB-Line will not include a hard-piped system from the hydrazine mononitrate source to the hydrazine mononitrate addition point.

6 The neptunium solution now in H-Canyon Tank 8.5 is the only solution that will be processed using the conclusions of this report, without further evaluation to ensure the conclusions are still valid.

$7 \quad$ The maximum hydrazine mononitrate concentration that will be specified by procedure for treatment of a process stream is $0.15 \mathrm{M}$.

8 The elutriant stream will be made up in one of the 600 -liter tanks $(\mathrm{CN}-22, \mathrm{CN}$ 23, CN-24, or CN-25).

9 If hydrazine mononitrate is added for the column adjustment stream in Cold Feed Prep, the column adjustment stream will be made up in one of the 600 liter tanks discussed in Assumption 8.

10 The column reconditioning stream and the decontamination wash stream will not be treated with hydrazine mononitrate.

\subsection{Discussion}

In this analysis, bounding assumptions are typically chosen to ensure the conclusions are robust. Examples are as follows:

- The temperature in the feed adjust tank was assumed to be $65^{\circ} \mathrm{C}$ when calculating the Henry's Law coefficient.

- The hydrazioc acid concentration can be up to $1 / 3$ of the hydrazine mononitrate concentration.

- The Cold Feed tanks with the largest volume were assumed when solutions were being prepared.

\subsection{Recommendations}

It is recommended that the assumptions made in arriving at the conclusion that an autocatalytic decomposition of hydrazoic acid is BEU be implemented in HB-Line. 


\subsection{References}

1. CBU-HCP-2003-00044, Rev. 0, HB-Line Np-237 Flowsheet, March 14, 2003

2. WSRC S1 Manual, Procedure OP4.25, Rev. 1, Chemical Control Program (U), $7 / 6 / 98$

3. DPST-59-648, "Recovery of Pu-238 and Np-237 from Irradiated NeptuniumAluminum Targets," revised 8/1/60

4. DP-808, "Uranium(IV) Nitrate as a Reducing Agent for Plutonium(IV) in the Purex Process," April 1963

5. SRT-ATS-2001-00003, Literature Search and Review of the Hazards of Hydrazoic Acid, Jan. 10, 2001

6. X-CLC-H-00146, Condensate Hydrazoic Acid Concentrations for Vapor from HB-Line Process Solutions, January 12, 2001

7. WSRC-TR-2003-00015, Rev. 0, Absorption of Hydrazoic Acid on Reillex ${ }^{\text {tm }}$ HPQ Anion Exchange Resin, February 2003

8. $\quad$ Not Used

9. W718118, Rev. 74, Cold Feed Prep P\&I Diagram, Partitioning Tanks, Process and Instruments

10. BPF 213457, Sheet 3, Vertical Tank

11. PV180694, Rev. 11, 75 Liter F/A Tank

12. Karraker, David G., Oxidation of Hydrazine by Nitric Acid, Inorg. Chem. 1985, 24, 4470-4477

13. Private Communication with David Karraker, SRTC, 7/18/03

14. FRM 221-HB-900D, Rev. 2, Cold Feed Prep 2 Calibration Charts, 1/29/04 